\title{
Quantifying the uncertainty in the ultimate recoverable oil reserves using the Monte Carlo simulation techniques from 'OWA' Marginal Field, Onshore Niger Delta, Nigeria
}

\author{
Olubunmi Christopher Adeigbe ${ }^{1}$, Isaac Folorunso Odedere ${ }^{2}$, \\ Omowunmi Idera Amodu ${ }^{1}$ \\ ${ }^{1}$ University of Ibadan, Department of Geology; Ibadan, Nigeria; \\ e-mail:olukris2009@gmail.com,omowunmiamodu93@gmail.com \\ ${ }^{2}$ Pan African University, Life and Earth Sciences Institute; Nigeria; e-mail:funso_odedere@yahoo.com
}

(C) 2018 Authors. This is an open access publication, which can be used, distributed and reproduced in any medium according to the Creative Commons CC-BY 4.0 License requiring that the original work has been properly cited.

Received: 9 May 2018; accepted: 15 October 2018; first published online: 26 November 2018

\begin{abstract}
A review on the development of marginal oil fields in Nigeria has now become an important strategic issue if it is to remain amongst the top producers in the global market, and these fields are vast, available all over the Niger Delta. One of the factors that makes a field marginal is the size of its reserves. Stochastic estimation gives a certainty in terms of the possible number of outcomes within the range of input parameters. In this work, four (4) deviated wells and 3D seismic volume (362 inlines and 401 traces) were interpreted for the evaluation of the field. The petrophysical evaluations were interpreted using the Power Log software and the Seismic, Geographix and Petrel softwares. Stochastic reserve estimation was done using Monte Carlo sampling techniques and subjected to uncertainty quantification using the Crystal Ball software by varying distributions and measuring sensitivity impact on the overall reserves. The production profile was predicted based on some assumptions and history matching which result in the overall Expected Ultimate Recovery (EUR).

The petrophysical analysis shows the reservoirs to be within the unconsolidated continental Benin Formation denoted as 'Intra-Benin' sands, an unconventional reservoir as supposed the normal reservoir rocks within the Agbada Formation. This indicated high porosity $(0.28)$, water resistivity $(7 \Omega \cdot m)$, and water saturation and also inferred Heavy Oil (low API). Nine hydrocarbon sands were identified but only three (B1, D and E), representing shallow, mid and deep reservoirs were further evaluated. $1 \mathrm{P}$ and $2 \mathrm{P}$ reserve estimates were $4.8 \mathrm{MMBO}$ and 5.7 MMBO for B1; 15.2 MMMscf and 16.4 MMMscf for D; 8.4 MMMscf and 8.8 MMMscf for E respectively. The Monte Carlo simulation of 1,000,000 trials with mainly triangular distribution assumption generated P10, P50, P90 were 6.5 MMBO, 5.6 MMBO and 4.4 MMBO for B1; 17.5 MMMscf, 13.7 MMMscf and 10.8 MMMscf for D; 10.4 MMMscf, $8 \mathrm{MMMscf}$ and 6.1 MMMscf for E respectively. The sensitivity impact of the input parameters were estimated and ranked, and the coefficient of variability ranges within $15 \%$ to $20 \%$ for the reservoirs indicating that there is a very low level uncertainty of reserve estimation around the P10, P50 and P90 percentiles which could be positive for investment decisions. 'OWA' marginal field reflects a typical low reserve (EUR) category found within the Niger Delta basin.
\end{abstract}

Keywords: marginal field, EUR, Monte Carlo, stochastic estimates, reserve

\section{INTRODUCTION}

The Niger Delta basin occupies the Gulf of Guinea continental margin in equatorial West Africa, between the latitude $3^{\circ}$ and $6^{\circ} \mathrm{N}$ and the longitude $5^{\circ}$ and $8^{\circ}$ E. and it is Nigeria and Africa's most prolific producing basin (Tuttle et al. 1999), with some untapped hydrocarbon reserves generally 
termed marginal. The Tertiary Niger Delta isdivided into three formations, from the youngest; Benin, Agbada and Akata formations, representing an overall prograding depositional facies that are distinguished mostly on the basis of sandshale ratios. The type sections of these formations are described in Short \& Stäuble (1967) and summarized in a variety of papers (Avbovbo 1978, Doust \& Omatsola 1990, Kulke 1995). The present study area falls within the Eastern Northern Depobelt part of the Niger Delta.

Marginal fields are associated with many challenges and thus subjected to immense risk analysis. A review on the development of marginal oil fields in Nigeria has now become an important strategic issue if it is to remain amongst the top producers in the global market, and these fields are vast within the Niger Delta (Fig. 1). A field can be considered to be a pool of hydrocarbon held within a structural or stratigraphic configuration whereas a marginal field is an oil field that may not produce enough net income to make it worth developing at a given time and/or which has not been exploited for long, due to factors such as: the size of its reserves, lack of nearby infrastructure or profitable consumers, high development costs, fiscal levies and technological constraints, environmental concerns, political stability, access and remoteness, and the price and price stability of the produced gas/liquid. However, should technical, local or economic conditions change; such fields can become commercial ones.

The marginal field program was introduced by the Federal Government to encourage indigenous participation in the strategic upstream sector of the oil industry. The program, which evolved from the Petroleum (Amendment) Decree Act No. 23 of 1996, was introduced to reduce the rate of abandonment of depleting oil fields by International Oil Companies (IOCs). Under the program, IOCs with Oil Mining Licences (OMLs) were required to farm-out oil fields to indigenous Exploration \& Production (E\&P) companies that were undeveloped for at least 10 years after discovery. In 2003, twenty four fields were awarded to 31 companies, with over $60 \%$ being onshore (Fig. 1).

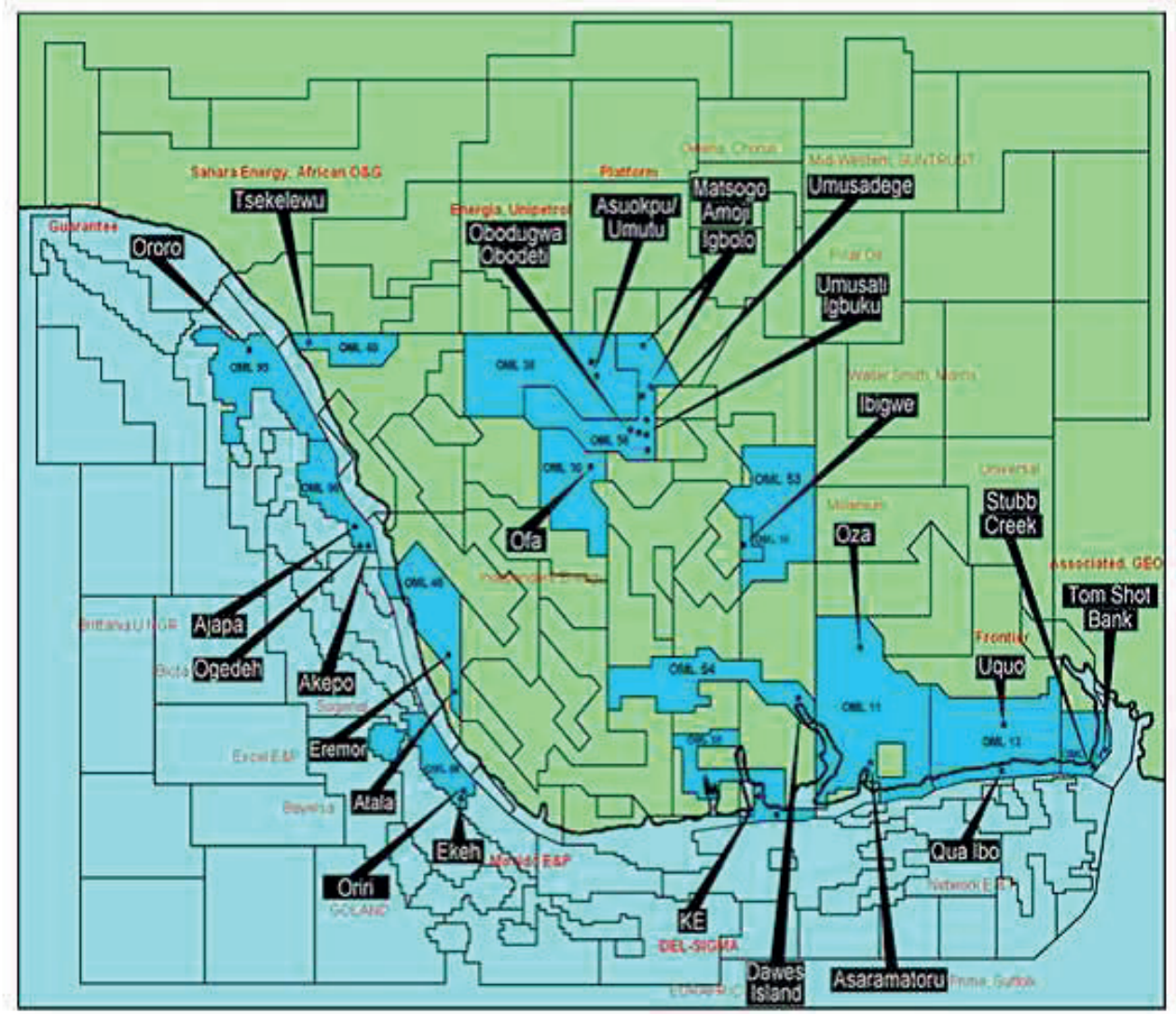

Fig. 1. 2003/2004 Niger-Delta marginal fields concession and their operators (https://goo.gl/images/UkdNB1) (Awotiku 2011) 
Ultimately Recoverable Resource (URR) is an estimate of the total amount of oil that will ever be recovered and produced from a field. In reserve estimation, deterministic and stochastic methods are used. In the deterministic method, a discrete value for each parameter is selected that is most appropriate for the corresponding resource category while in the stochastic method, the estimator defines a distribution representing the full range of possible values for each input parameter. Uncertainty quantification creates value only to the extent that it holds the possibility of changing a decision that would otherwise be made differently (Bickel \& Bratvold 2008).

\section{METHODOLOGY}

This research involves the application of Monte Carlo simulation techniques to quantify the uncertainty in recovering ultimately the oil resources from 'OWA' marginal field in the eastern part of the Niger Delta basin. The data used for this research work are secondary data obtained through the Department of Petroleum Resources (DPR), Nigeria. All data were integrated to evaluate the field volumetrically and the corresponding reports and interpretation were used in quantifying the risk and uncertainties.

\section{Data}

Four (4) deviated wells and 3D seismic volume (362 inlines and 401 traces) were interpreted for the evaluation of the field. The checkshot data for well 1 which was shared for the other two wells was used to generate a velocity function which gave the conversion to the depth structural map.

\section{Procedures}

The petrophysical evaluations were interpreted using the Power Log software and the seismic evaluation, Geographix and Petrel software. These values were then used in computing the volumetrics:

$$
\begin{aligned}
& \text { STOOIP }=\frac{7758 \times \mathrm{NTG} \times \text { POROSITY } \times\left(1-S_{w}\right) \times \mathrm{GRV}}{\mathrm{FVF}}, \\
& \mathrm{GIIP}=\frac{43560 \times \mathrm{NTG} \times \mathrm{POROSITY} \times\left(1-S_{w}\right) \times \mathrm{GRV}}{\mathrm{FVF}},
\end{aligned}
$$

Reserve $=\mathrm{STOOIP} \times \mathrm{RF}$, where:

NTG - Net to Gross,

$S_{w}$ - water saturation,

GRV - Gross Rock Volume (Area $\times$ Height),

FVF - Formation Volume Factor,

RF - Recovery Factor.

Then, the deterministic estimates, 1P, 2P, 3P of the volumes were calculated using a single base (average) value for each parameter. The Monte Carlo simulation (Crystal Ball) approach was then used for the stochastic estimates by varying distributions and measuring sensitivity impact on the overall reserves.

\section{RESULTS AND DISCUSSION}

Nine hydrocarbon sands (corresponding to low gamma ray readings and high resistivity), of which only three (3); B1, D and E reservoirs (representing shallow, mid and deep reservoirs) were further evaluated, were identified by interpreting (Tab. 1) and correlating the logs across the four wells (Figs. 2-5). All parameters especially those related to volumetrics were quantified using the programmed calculations on the software (Tabs. 2-4).

Table 1

Qualitative interpretation of the reservoirs

\begin{tabular}{|l|l|c|c|c|}
\hline \multicolumn{2}{|l|}{ Reservoir } & B1 & D & E \\
\hline Fluid & oil & gas & gas \\
\hline \multicolumn{2}{|l|}{ HWC [ft] } & -5506 & -5728 & $-6754(\mathrm{GDT})$ \\
\hline \multirow{2}{*}{ Well 1 } & top [ft] & 5460 & 5677 & 6713 \\
& base [ft] & 5514 & 5745 & 6748 \\
\hline \multirow{2}{*}{ Well 2 } & top [ft] & 5467 & 5673 & 6711 \\
& base [ft] & 5518 & 5742 & 6734 \\
\hline \multirow{2}{*}{ Well 3 } & top [ft] & 5467 & 5681 & 6723 \\
& base [ft] & 5517 & 5747 & 6754 \\
\hline
\end{tabular}

One major fault transcended the whole seismic volume and a total of six normal faults were mapped. The faults are listric faults typical of the Niger Delta Basin, i.e, faults that flatten with depth (Fig. 6). They are all synthetic faults, formed during deposition, and their direction of dip was towards the south. Time Slicing was used to QC (Quality Control) the fault and structural mapping. This enhanced the visibility of the plane intersection of the faults at different times. The alignment and discontinuity on the time slice (Fig. 7) surface gave the inference of where the faults intersect the plane. 


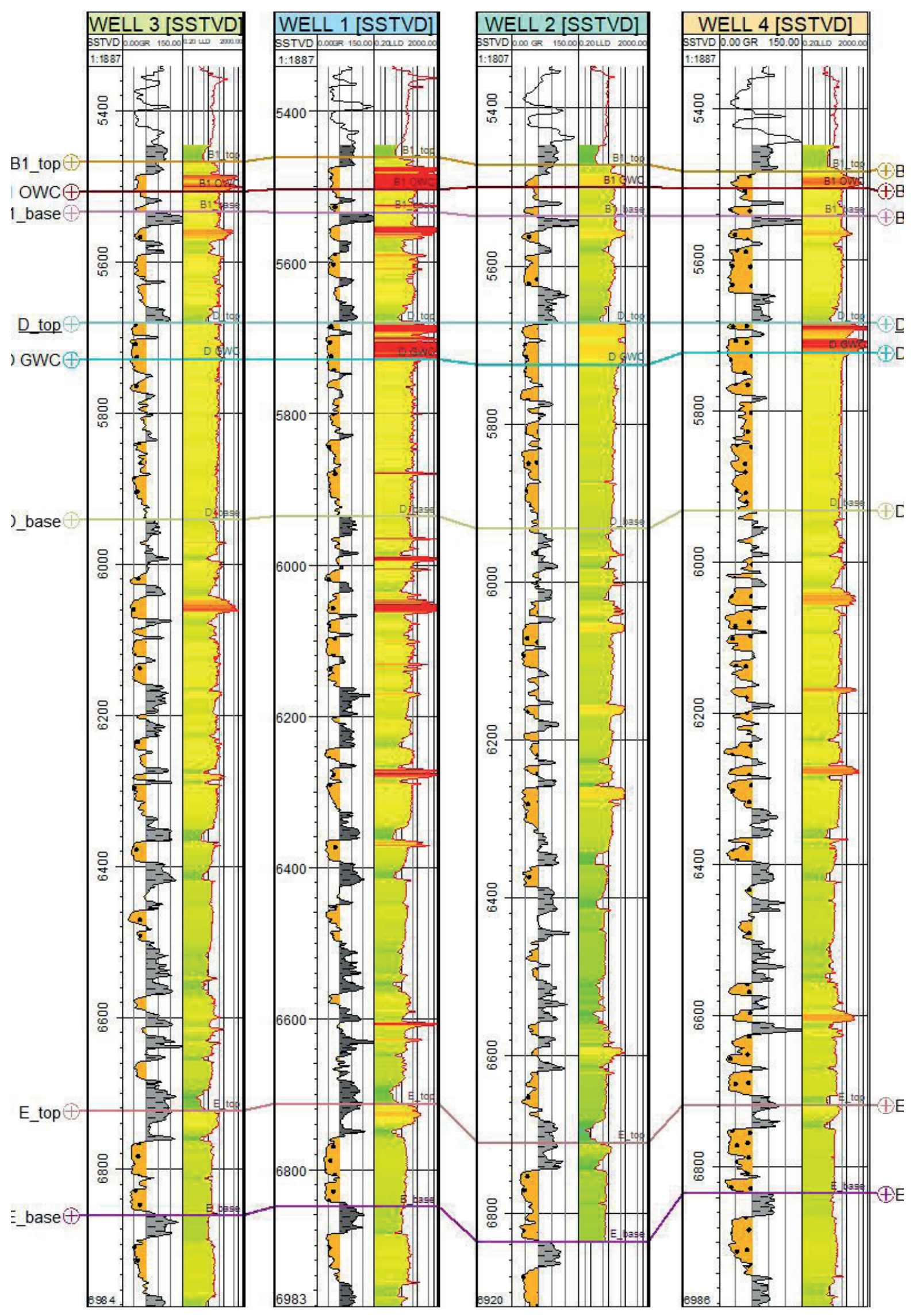

Fig. 2. Well correlation [ft] (flattened at surface) 


\begin{tabular}{|c|c|c|c|}
\hline$\stackrel{5}{\mathrm{Z}}$ & बे. & $\left|\begin{array}{c}\overrightarrow{0} \\
0 \\
0\end{array}\right|$ & $\stackrel{\infty}{\circ}$ \\
\hline 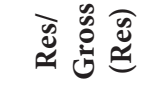 & $\begin{array}{c}\infty \\
\infty \\
\infty \\
0 \\
0\end{array}$ & 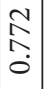 & \begin{tabular}{l} 
\\
\multirow{2}{0}{} \\
0
\end{tabular} \\
\hline 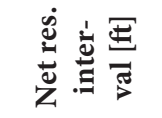 & $\begin{array}{l}\stackrel{\infty}{\infty} \\
\stackrel{+}{+}\end{array}$ & 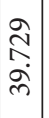 & $\begin{array}{l}\hat{\hat{o}} \\
\stackrel{0}{\mathrm{~m}}\end{array}$ \\
\hline 高总部 & $\begin{array}{l}0 \\
0 \\
0 \\
0 \\
0\end{array}$ & 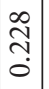 & \begin{tabular}{l}
$\overrightarrow{5}$ \\
\multirow{0}{0}{}
\end{tabular} \\
\hline 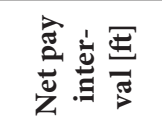 & $\begin{array}{l}\vec{\partial} \\
\infty \\
\dot{j}\end{array}$ & $\begin{array}{l}\underset{4}{+} \\
\stackrel{+}{二}\end{array}$ & $\begin{array}{l}\overrightarrow{1} \\
\infty \\
\stackrel{2}{\text { in }}\end{array}$ \\
\hline 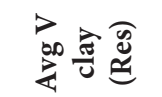 & $\begin{array}{l}\hat{a} \\
\text { Oे } \\
\dot{0}\end{array}$ & 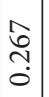 & 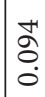 \\
\hline 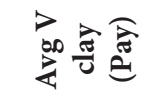 & $\begin{array}{l}0 \\
\infty \\
0 \\
0 \\
0\end{array}$ & $\mid$ & ôे \\
\hline 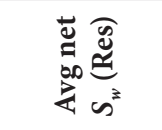 & ণ্రి & 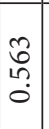 & 年 \\
\hline 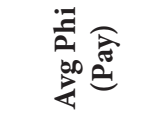 & $\stackrel{m}{0}$ & 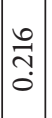 & $\hat{\widehat{c}}$ \\
\hline 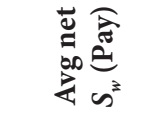 & $\begin{array}{l}\infty \\
\infty \\
0 \\
0\end{array}$ & $\hat{m}$ & సे \\
\hline 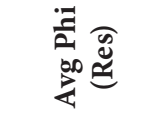 & ते & 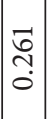 & 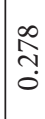 \\
\hline 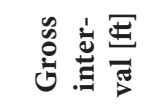 & 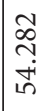 & $\left|\begin{array}{c}\hat{N} \\
\text { fr } \\
\dot{i n}\end{array}\right|$ & 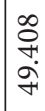 \\
\hline 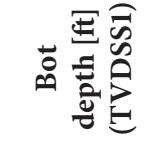 & 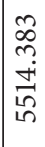 & $\left|\begin{array}{c}0 \\
0 \\
0 \\
0 \\
0 \\
1 \\
n \\
1\end{array}\right|$ & 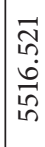 \\
\hline 苍 & $\begin{array}{l}\infty \\
\infty \\
: \\
1 \\
1\end{array}$ & $\begin{array}{l}\infty \\
0 \\
0 \\
1 \\
1\end{array} \mid$ & $\underset{i n}{i}$ \\
\hline 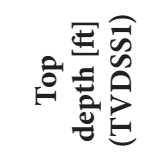 & $\begin{array}{l}\infty \\
\infty \\
\text { ñ. } \\
0 \\
0 \\
\text { in }\end{array}$ & $\begin{array}{l}\hat{0} \\
0 \\
0 \\
0 \\
0 \\
1 \\
1\end{array}$ & 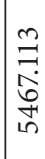 \\
\hline 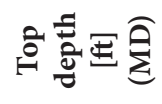 & ڤ్ర & $\left|\begin{array}{l}\tilde{N} \\
\hat{D} \\
i\end{array}\right|$ & $\begin{array}{l}\text { Oे. } \\
\text { in }\end{array}$ \\
\hline 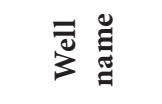 & $\overrightarrow{\bar{m}}$ & $\begin{array}{c}\stackrel{\sim}{\vec{*}} \\
\stackrel{\sim}{3}\end{array}$ & $\frac{m}{\vec{*}}$ \\
\hline
\end{tabular}

\begin{tabular}{|c|c|c|c|}
\hline $\overrightarrow{\mathrm{Z}}$ & ฉి & âे & 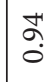 \\
\hline 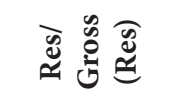 & 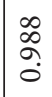 & \begin{tabular}{l}
$\infty$ \\
\multirow{2}{0}{} \\
0 \\
0
\end{tabular} & 令 \\
\hline 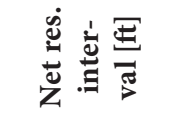 & $\begin{array}{l}\text { â. } \\
\text { శิ }\end{array}$ & 突 & $\begin{array}{l}\infty \\
\infty \\
\stackrel{0}{+} \\
\dot{f}\end{array}$ \\
\hline 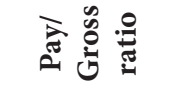 & $\begin{array}{l}\text { ô } \\
0 \\
0\end{array}$ & 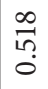 & 且 \\
\hline 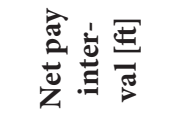 & 务 & $\begin{array}{l}\infty \\
\vdots \\
\vdots \\
\infty \\
n \\
m\end{array}$ & $\begin{array}{l}a \\
\dot{a} \\
\dot{m}\end{array}$ \\
\hline 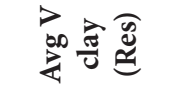 & \begin{tabular}{l}
0 \\
\multirow{1}{0}{} \\
0 \\
0
\end{tabular} & $\begin{array}{l}\vec{n} \\
0 \\
0\end{array}$ & $\stackrel{\widetilde{\Xi}}{0}$ \\
\hline 忞忩 & 命 & $\begin{array}{l}\stackrel{n}{0} \\
\hat{o} \\
0\end{array}$ & तี \\
\hline 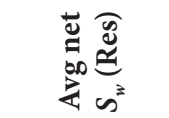 & $\begin{array}{l}\overrightarrow{7} \\
\text { ठ }\end{array}$ & $\begin{array}{l}\stackrel{2}{2} \\
\stackrel{0}{0} \\
0\end{array}$ & ने \\
\hline 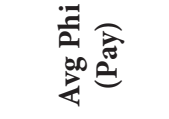 & 命 & 文 & đָ \\
\hline 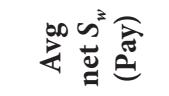 & 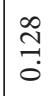 & $\begin{array}{l}\hat{0} \\
1 \\
0 \\
0\end{array}$ & 辛 \\
\hline 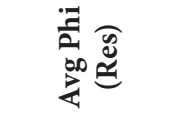 & 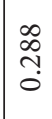 & 节 & 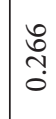 \\
\hline 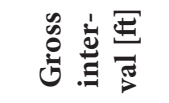 & $\underset{\substack{\infty \\
\infty \\
\infty}}{\stackrel{\infty}{0}}$ & $\begin{array}{l}\infty \\
10 \\
4 \\
\vdots \\
6\end{array}$ & 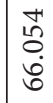 \\
\hline 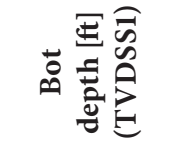 & $\begin{array}{l}\text { th } \\
\text { ले } \\
\text { ind } \\
\text { in } \\
\text { in }\end{array}$ & \begin{tabular}{|l}
$\vec{\infty}$ \\
$\stackrel{7}{j}$ \\
$\mathbb{H}$ \\
in
\end{tabular} & $\begin{array}{l}\text { a } \\
5 \\
b \\
0 \\
1 \\
\text { in }\end{array}$ \\
\hline 苍营玉完 & के & 余 & i⿱ \\
\hline 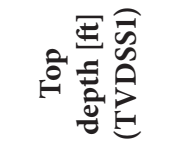 & $\begin{array}{l}\overrightarrow{1} \\
\infty \\
0 \\
0 \\
b \\
i n \\
i n\end{array}$ & 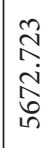 & $\begin{array}{l}2 \\
\infty \\
\infty \\
\infty \\
\infty \\
0 \\
i n\end{array}$ \\
\hline 各 & $\underset{\substack{\infty \\
i n}}{i n}$ & $\begin{array}{l}\infty \\
\infty \\
\infty \\
n \\
n\end{array}$ & $\begin{array}{l}\infty \\
\infty \\
\infty \\
i n\end{array}$ \\
\hline 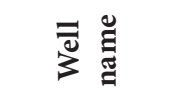 & $\overline{\bar{\nu}}$ & $\stackrel{2}{\bar{*}}$ & $\frac{m}{7}$ \\
\hline
\end{tabular}

\begin{tabular}{|c|c|c|c|}
\hline$\stackrel{\varphi}{\mathrm{Z}}$ & {$\left[\begin{array}{c}L \\
\infty \\
0 \\
0\end{array}\right.$} & $\hat{o}$ & în \\
\hline 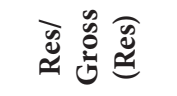 & $\begin{array}{l}\tilde{0} \\
\hat{0} \\
0 \\
0\end{array}$ & $\begin{array}{l}\vec{b} \\
\text { है } \\
0\end{array}$ & $\begin{array}{l}\infty \\
i n \\
0 \\
0\end{array}$ \\
\hline 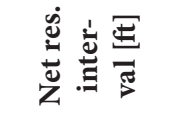 & $\begin{array}{l}\infty \\
2 \\
i \\
\text { ì }\end{array}$ & $\stackrel{\partial}{=}$ & $\stackrel{\substack{n\\
\\
}}{=}$ \\
\hline 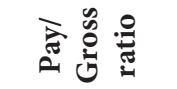 & $\begin{array}{l}\infty \\
\sum_{n} \\
0 \\
0\end{array}$ & in & F \\
\hline 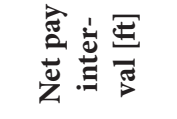 & $\begin{array}{l}\alpha \\
\partial \\
\infty \\
-1\end{array}$ & $\stackrel{g}{=}$ & 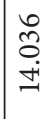 \\
\hline 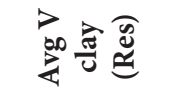 & 竎 & $\begin{array}{l}n \\
\text { nn } \\
0 \\
0\end{array}$ & I \\
\hline 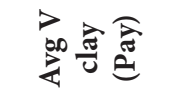 & 於 & 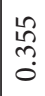 & 今̂ \\
\hline 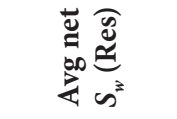 & 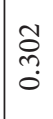 & I্ & ồ \\
\hline 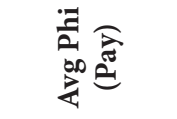 & 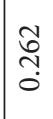 & $\stackrel{\infty}{\circ}$ & तु \\
\hline 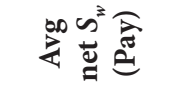 & $\vec{j}$ & ఫ్ & $\vec{m}$ \\
\hline 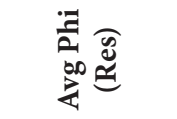 & 空 & $\stackrel{\infty}{0}$ & $\stackrel{1}{\sim}$ \\
\hline 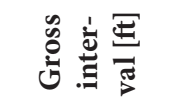 & 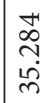 & $\begin{array}{l}\vec{\infty} \\
+ \\
\stackrel{\sim}{\sim}\end{array}$ & 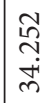 \\
\hline 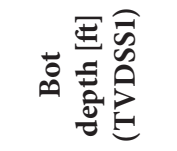 & 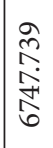 & $\begin{array}{l}\infty \\
\infty \\
+1 \\
\tilde{d} \\
6\end{array}$ & $\begin{array}{l}\tilde{2} \\
\hat{0} \\
\hat{N} \\
\hat{N} \\
0\end{array}$ \\
\hline 总劳玉完 & สิ & 这 & $\stackrel{\infty}{\infty}$ \\
\hline 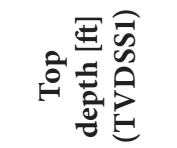 & $\begin{array}{l}\text { I } \\
\stackrel{1}{1} \\
\text { î }\end{array}$ & $\begin{array}{l}\text { 2. } \\
2 \\
0 \\
0 \\
\hat{1} \\
0\end{array}$ & 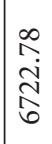 \\
\hline 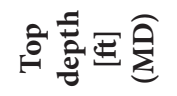 & $\mid \begin{array}{l}1 \\
\infty \\
0 \\
0\end{array}$ & $\begin{array}{c}\hat{1} \\
\substack{0 \\
0}\end{array}$ & F \\
\hline 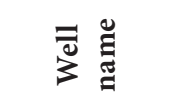 & $\overrightarrow{\bar{\sigma}}$ & $\frac{1}{7}$ & $\begin{array}{l}m \\
\overline{0} \\
3\end{array}$ \\
\hline
\end{tabular}




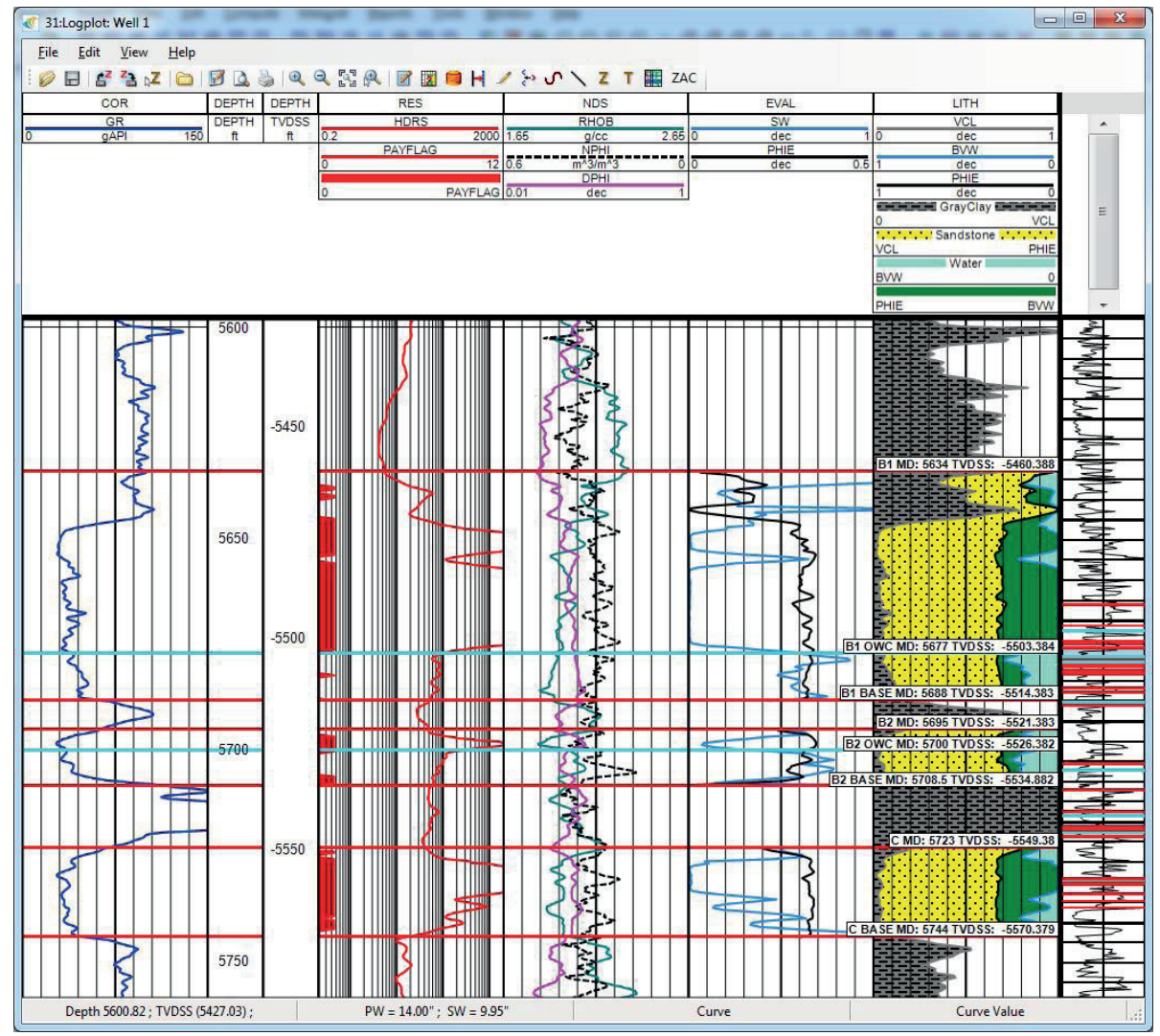

Fig. 3. Sand B1

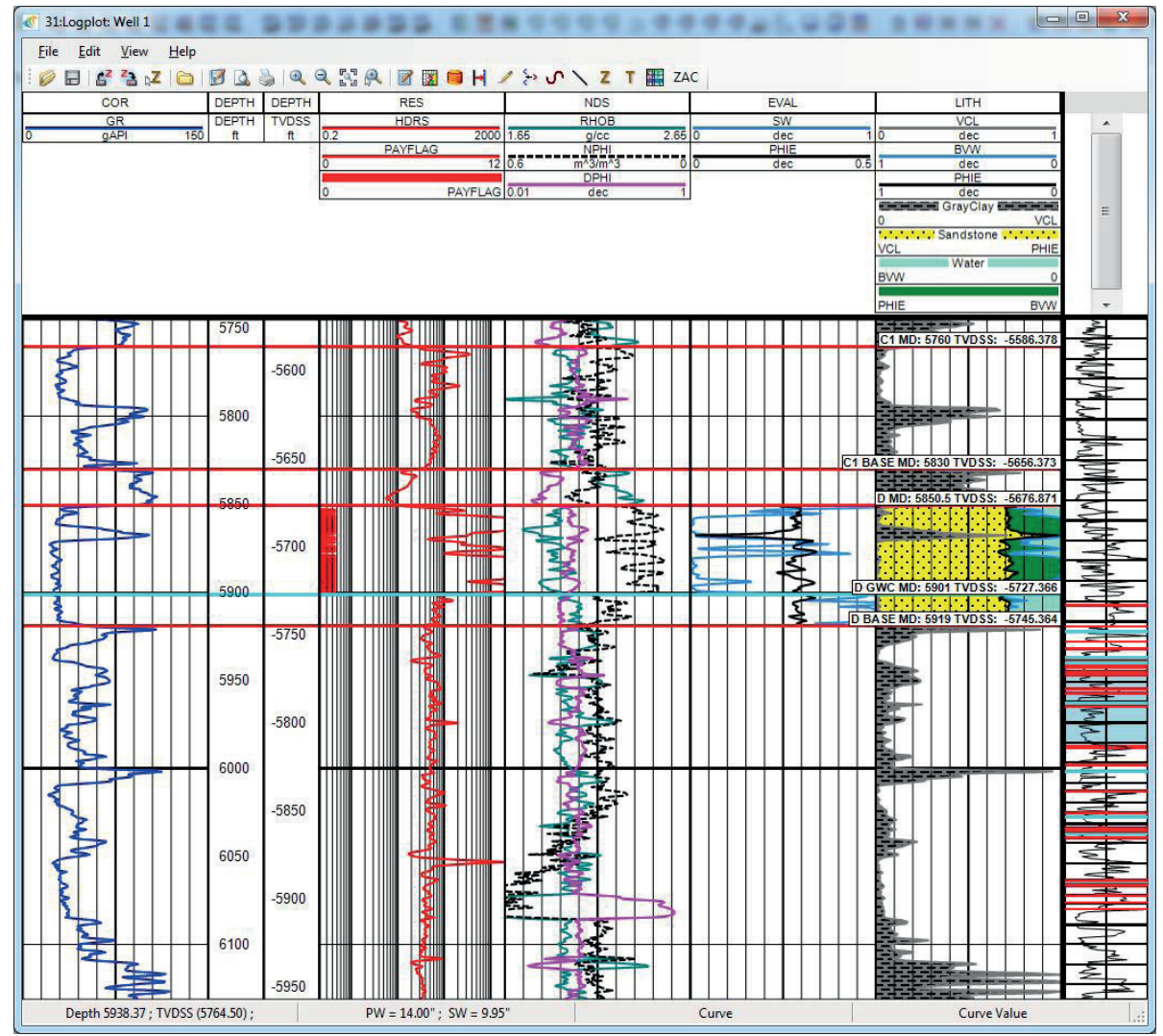

Fig. 4. Sand D 


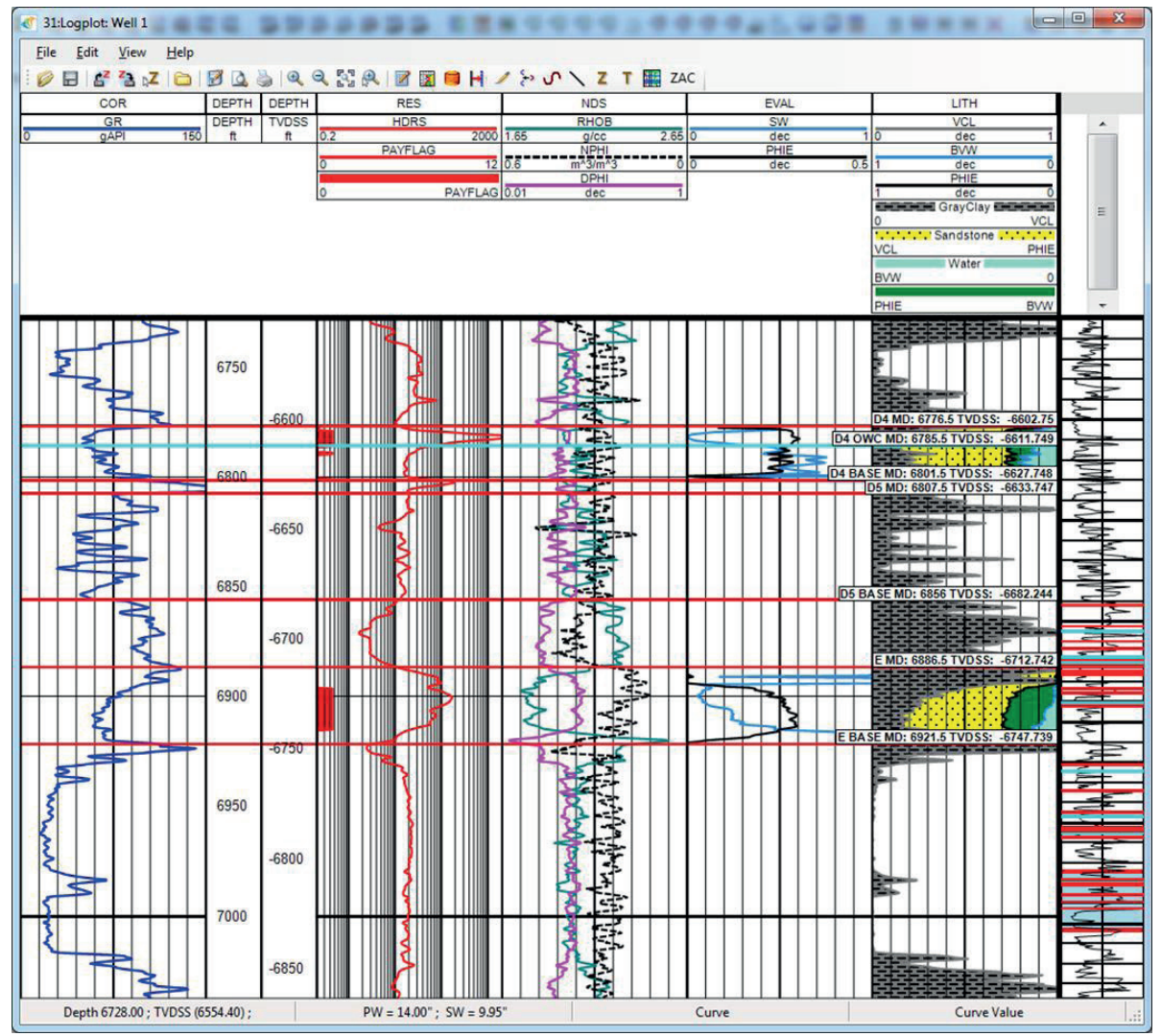

Fig. 5. Sand $E$

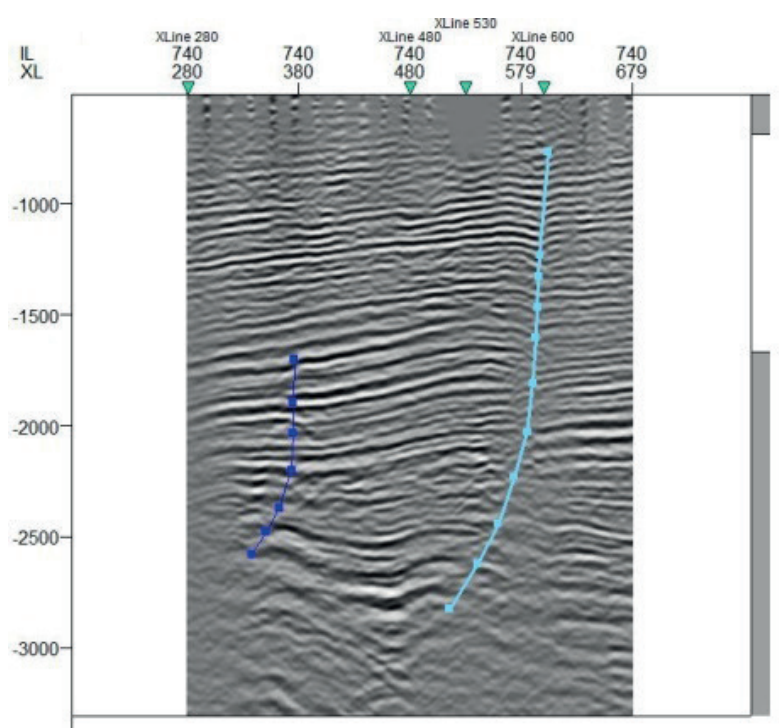

Fig. 6. Interpreted faults, in time

Three levels (B1, D, and E) representing a part of the shallow, mid, and deeper horizons for the general field configuration were mapped (Fig. 8) out of the nine identified hydrocarbon sands. A careful correlation of the lines and traces through the

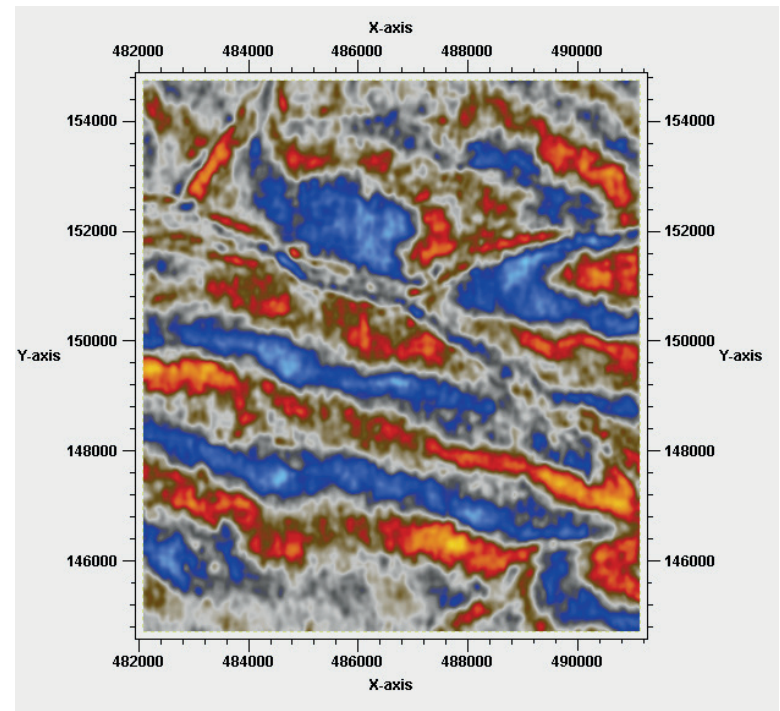

Fig. 7. Time slice at $1600 \mathrm{~ms}$

whole seismic volume was made at an interval of ten lines (plane step), which eventually generated the time-structured maps. The velocity function derived from the checkshot was used in converting to the depth structured maps (Fig. 9). 


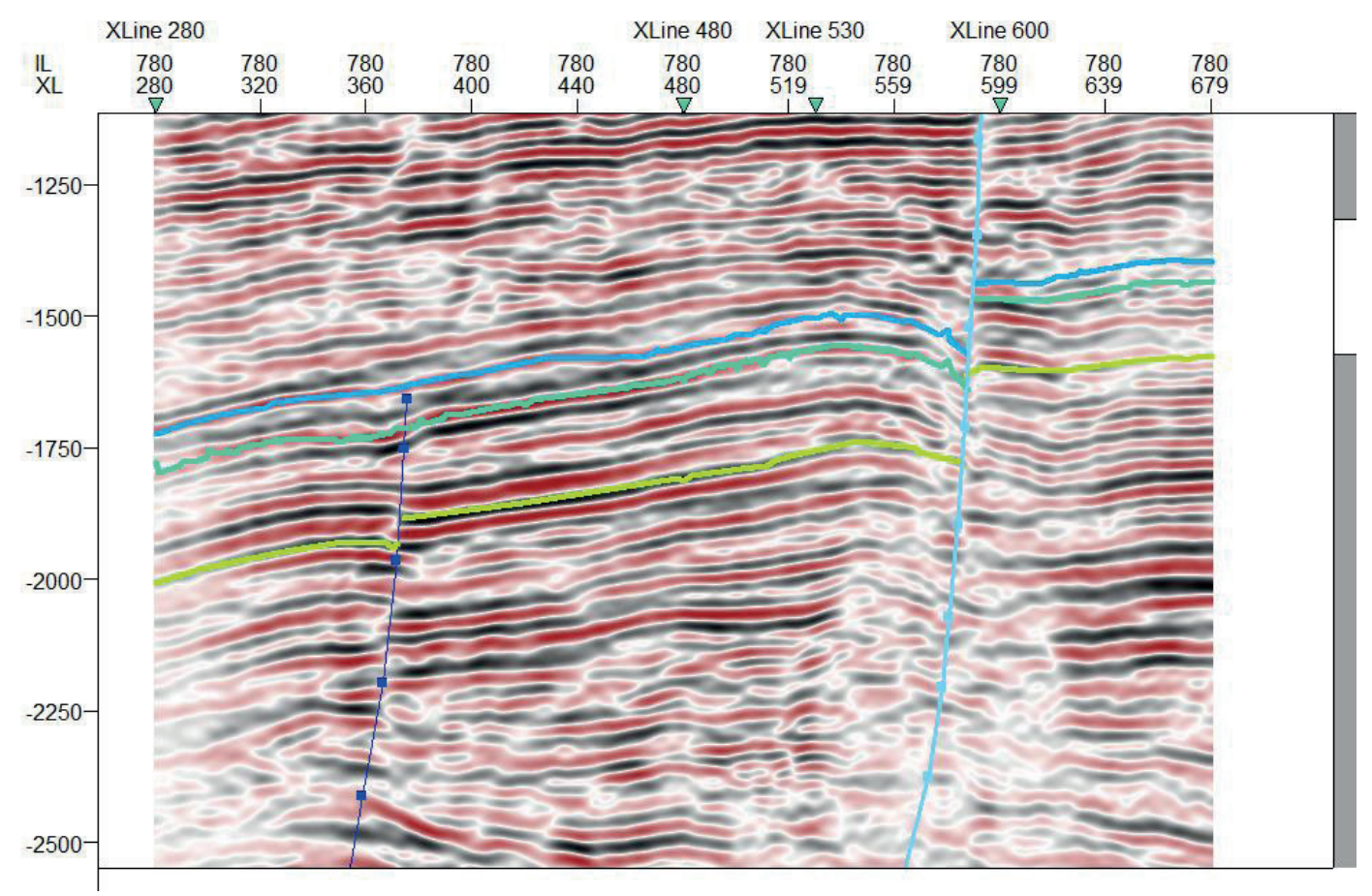

Fig. 8. Interpreted horizons, in time

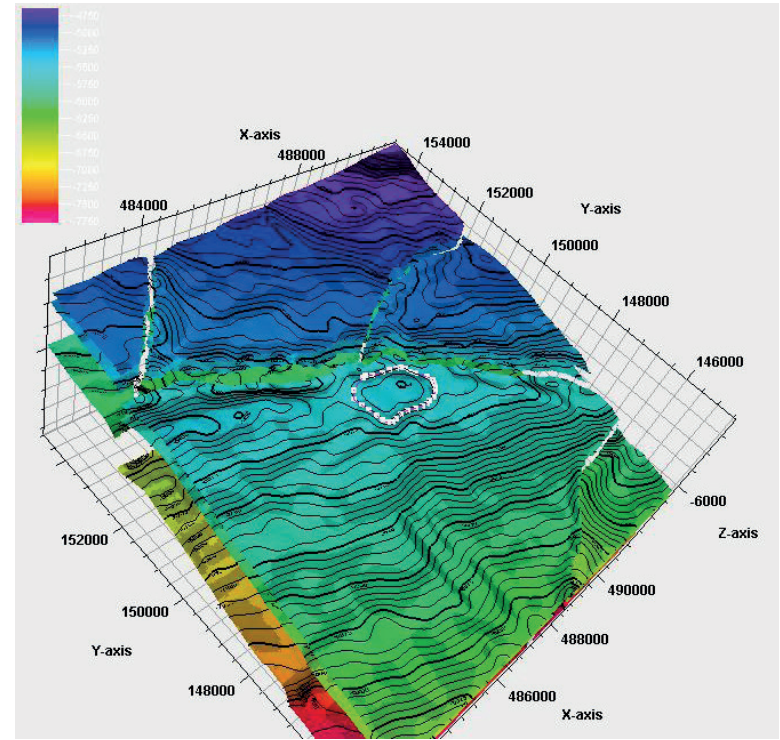

Fig. 9. Interpreted surfaces: depth structure maps [ft]

\section{Volumetrics}

As provided from the petrophysical evaluations, the parameters for the volumetrics $\left(S_{w}\right.$, porosity, NTG) were used in the Stock Tank Original Oil in Place (STOOIP), Gas Initially in Place (GIIP) equation and in the calculation of the field reserve from the three wells. The GRV was also estimated from the depth structural maps. The FVF for gas was assumed to be 0.004 while for oil as 1.45 . The $\mathrm{RF}$ used was 35\% assuming if production will not be induced or enhanced.

\section{Deterministic estimates}

A single outcome of recoverable quantities is derived for each deterministic increment or scenario (Tab. 5):

$1 \mathrm{P}=\mathrm{P} 1,2 \mathrm{P}=\mathrm{P} 1+\mathrm{P} 2,3 \mathrm{P}=\mathrm{P} 1+\mathrm{P} 2+\mathrm{P} 3$,

where $\mathrm{P} 1, \mathrm{P} 2$, and $\mathrm{P} 3$ are denoted proven, probable and possible reserves respectively. $\mathrm{P} 1$ is the reserve penetrated by the well path, the hydrocarbon column thickness from the impact point seen by the well. $\mathrm{P} 2$ is the reserve from the impact point to the crest of the reservoir while the P3, if the Hydrocarbon Water Contact (HWC) wasn't seen, is technically estimated to the 'spill point' of the structure (closure) or the thickness of the P1 well path is halved. If the HWC is seen, P2 and P3 are taken to be equal. The deterministic estimates averagely fall within the Egbogah (2011) classification, based on surface terrain and the typical range of minimal recoverable reserves required for profitable development of the marginal fields in the Niger Delta (Tab. 6). 
Table 5

Deterministic estimates of the reservoirs

\begin{tabular}{|l|c|c|c|}
\hline \multicolumn{1}{|c|}{ Reservoir } & 1P & 2P & 3P \\
\hline B1 [MMBO] & 4.8 & 5.7 & 5.7 \\
\hline D [MMMscf] & 15.2 & 16.4 & 16.4 \\
\hline E [MMMscf] & 8.4 & 8.8 & 12.6 \\
\hline
\end{tabular}

Table 6

Categories of marginal fields (Egbogah 2011)

\begin{tabular}{|c|c|c|}
\hline Category & $\begin{array}{c}\text { Recoverable oil } \\
{[\text { MMSTB] }}\end{array}$ & Terrain \\
\hline 1 & $2-10$ & onshore land \\
\hline 2 & $7-20$ & onshore swamp \\
\hline 3 & $12-25$ & coastal offshore \\
\hline 4 & $20-45$ & $\begin{array}{c}\text { continental shelf } \\
\text { offshore }\end{array}$ \\
\hline 5 & $>40$ & deep offshore \\
\hline
\end{tabular}

\section{Stochastic estimates}

Stochastic estimates differ only from probabilistic ones in that they are derived from constrained knowledge or analogue information. The percentile rank for oil and gas estimates, unlike the common usage, is in the reverse order. P90 denotes the highest level of confidence i.e. at least $90 \%$ of the range of volume that will be obtained will not be lower than the P90 value while P10 is the lowest level of confidence i.e. at least $10 \%$ of the range of volume that will be obtained will not be lower than the P10 value. The Monte Carlo simulation randomly selects available data within the range of assumptions or distributions to forecast values (Awotiku 2011). In this case, the parameters were assumed within the triangular distribution giving the parameters a loop that fall within known estimations i.e., the evaluation from the three wells. The smallest value from the three wells was used as 'minimum', the base or mid value for the 'likeliest' and the largest value for the 'maximum'.

$\mathrm{P} 10$, P50, P90 were 6.5 MMBO, 5.6 MMBO and 4.4 MMBO for B1; 17.5 MMMscf, 13.7 MMMscf and 10.8 MMMscf for D; 10.4 MMMscf, 8 MMMscf and 6.1 MMMscf for E respectively (Figs. 10-12). The coefficient of variability is $15 \%$, $19 \%$ and $20 \%$ for B1, D and E respectively. The coefficient of variation must be generally low for less risk and high confidence. These values of the coefficient of variation indicated that there is a very low level uncertainty of reserve estimation based on the distributions of input parameters.

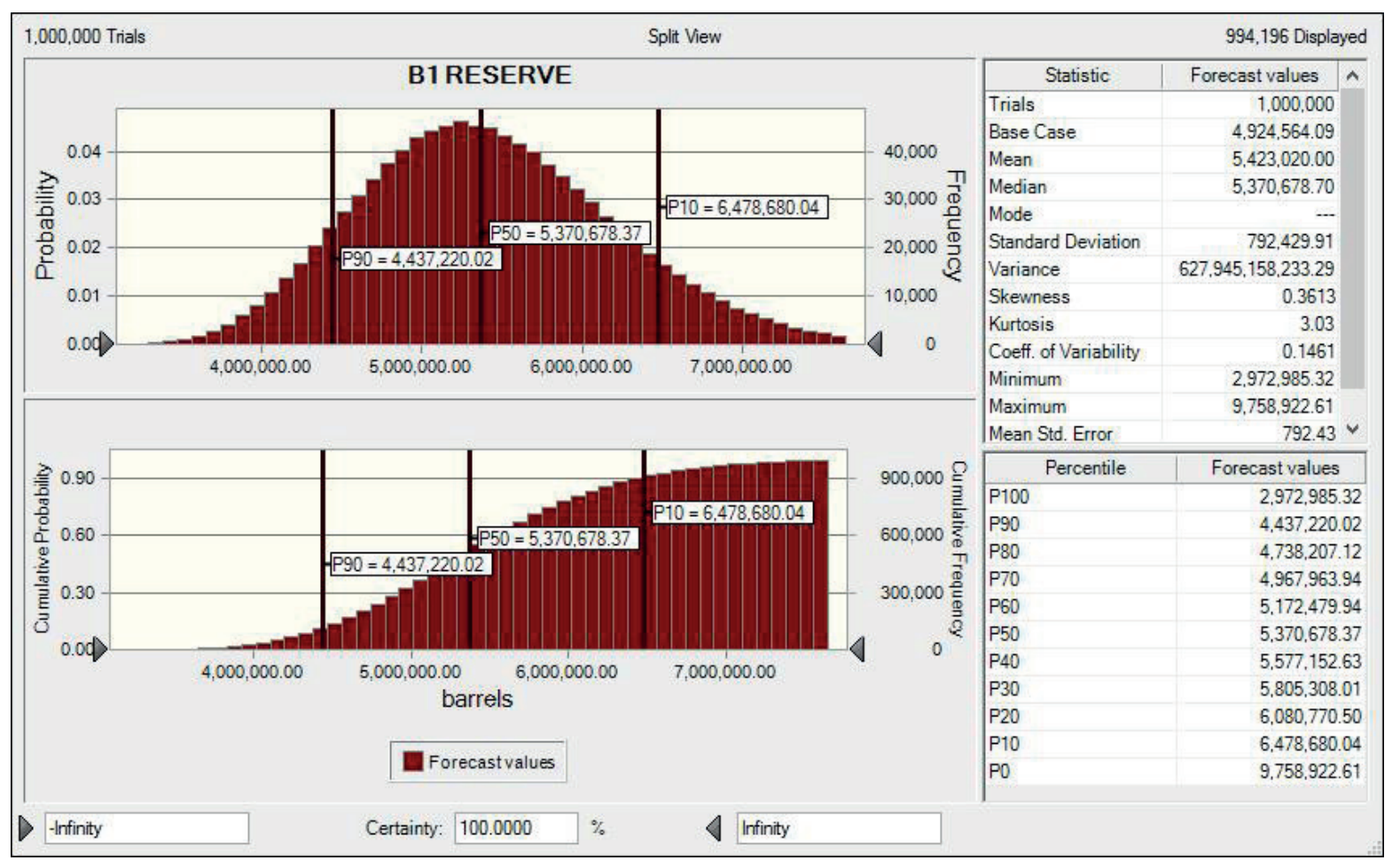

Fig. 10. B1 reserve simulation 


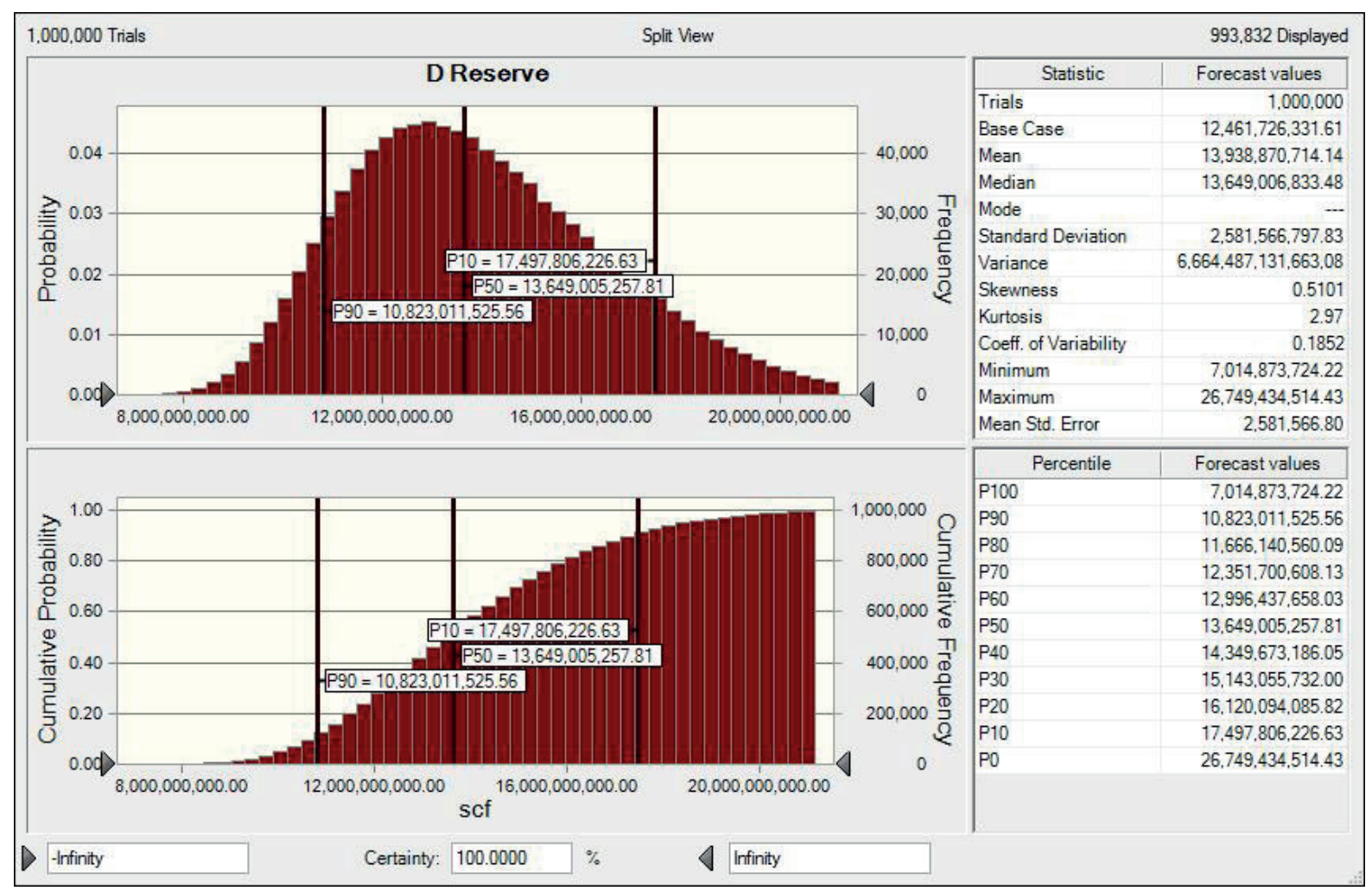

Fig. 11. D reserve simulation

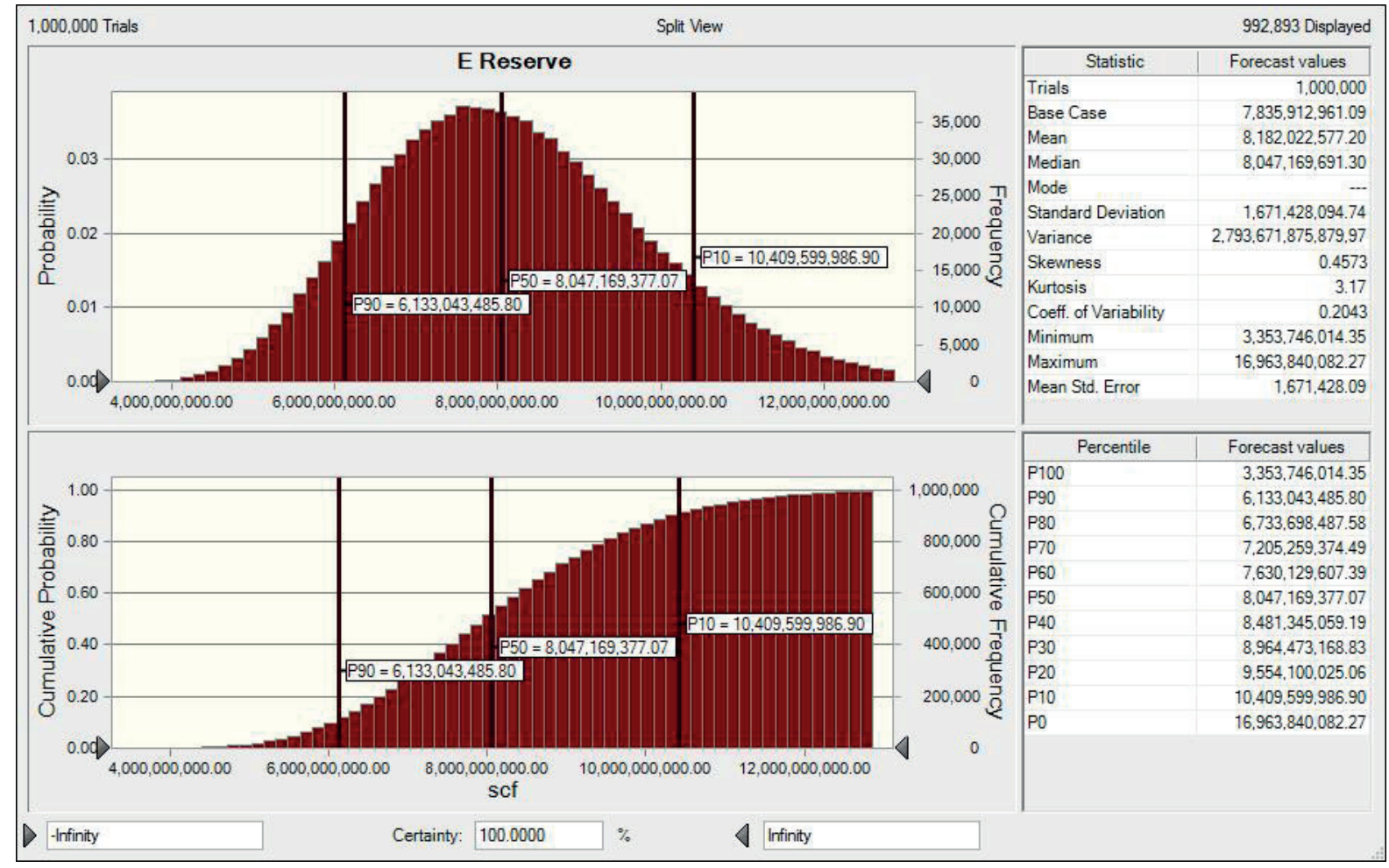

Fig. 12. E reserve simulation 
The sensitivity analysis is a measure of the effect of the different input parameters on the simulation (Figs. 13-15). A change in the highest sensitivity impact will cause a great effect on the overall reserve simulation as in the case of $40.6 \%$ of GRV in B1 reserve and $69.6 \%$ of $S_{h}$ in D reserve.

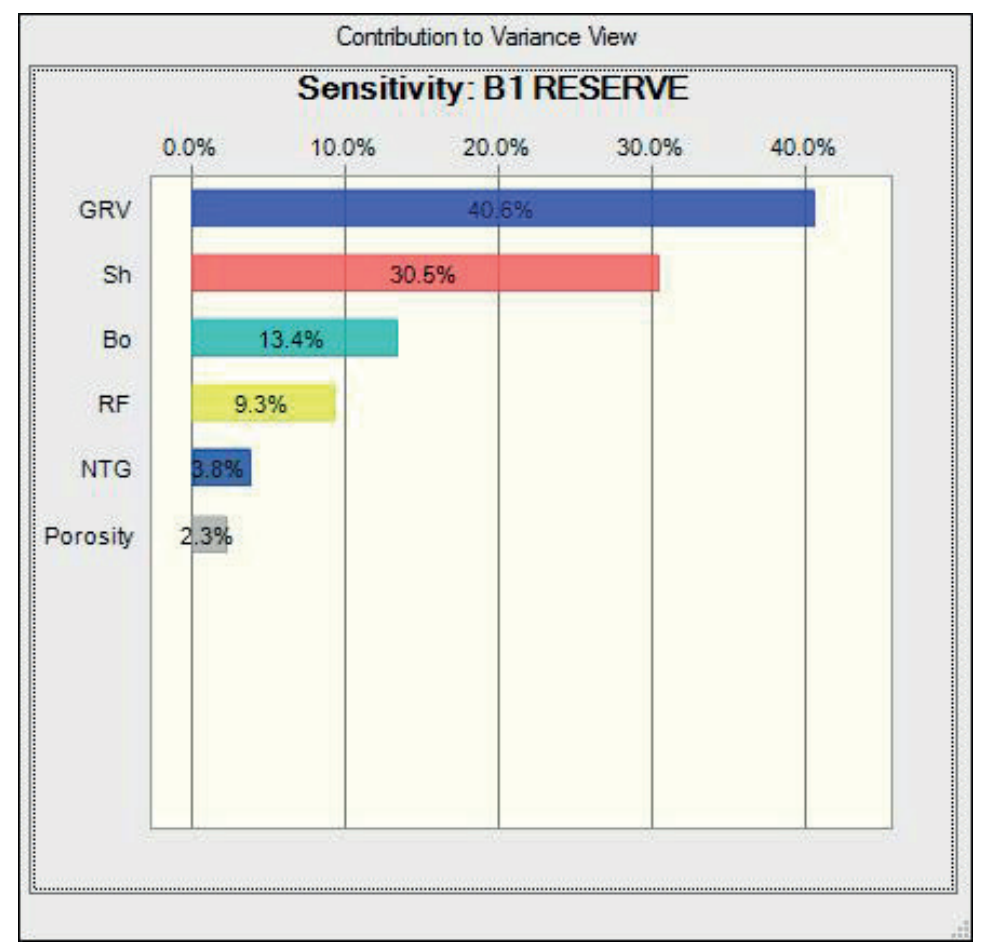

Fig. 13. The sensitivity impact on the B1 reserve simulation. Explanations: GRV - Gross Rock Volume, Sh (1 - Sw) - hydrocarbon saturation, Bo-formation volume factor (oil), NTG - Net to Gross, RF - Recovery Factor

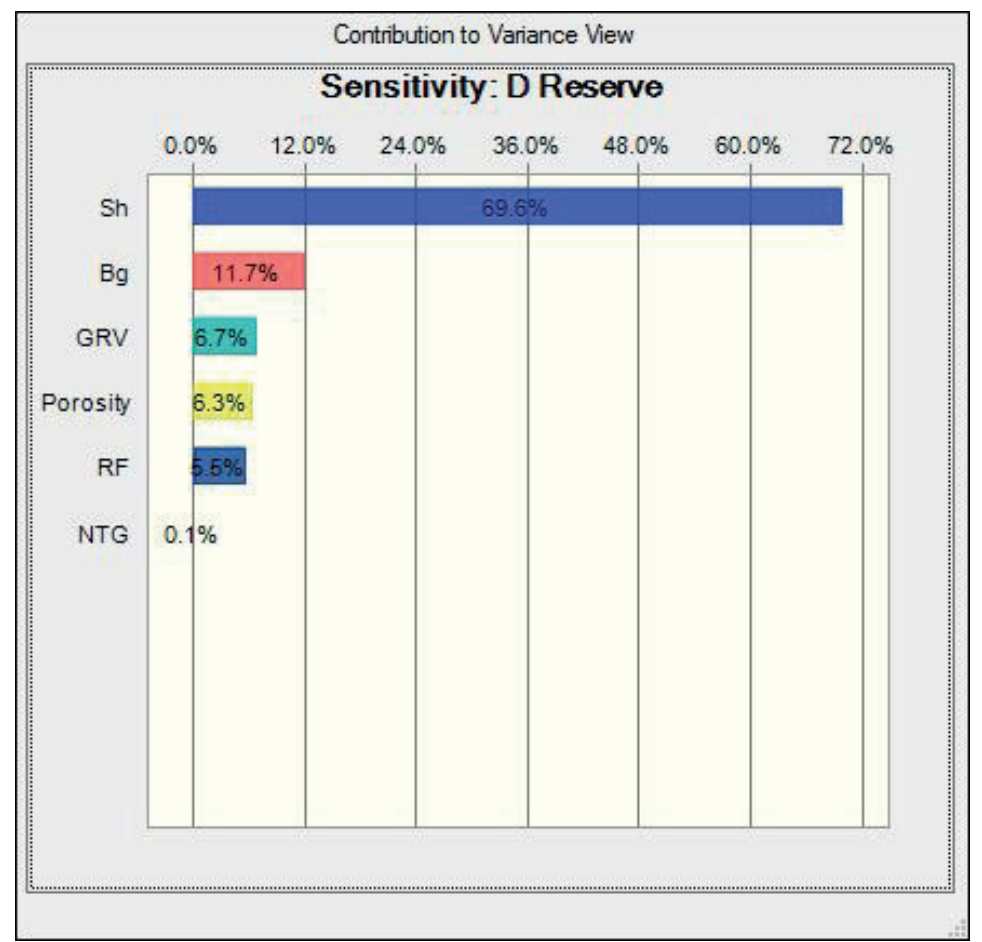

Fig. 14. The sensitivity impact on the D reserve simulation. Explanations: Bg - formation volume factor (gas), others - as in Figure 13 


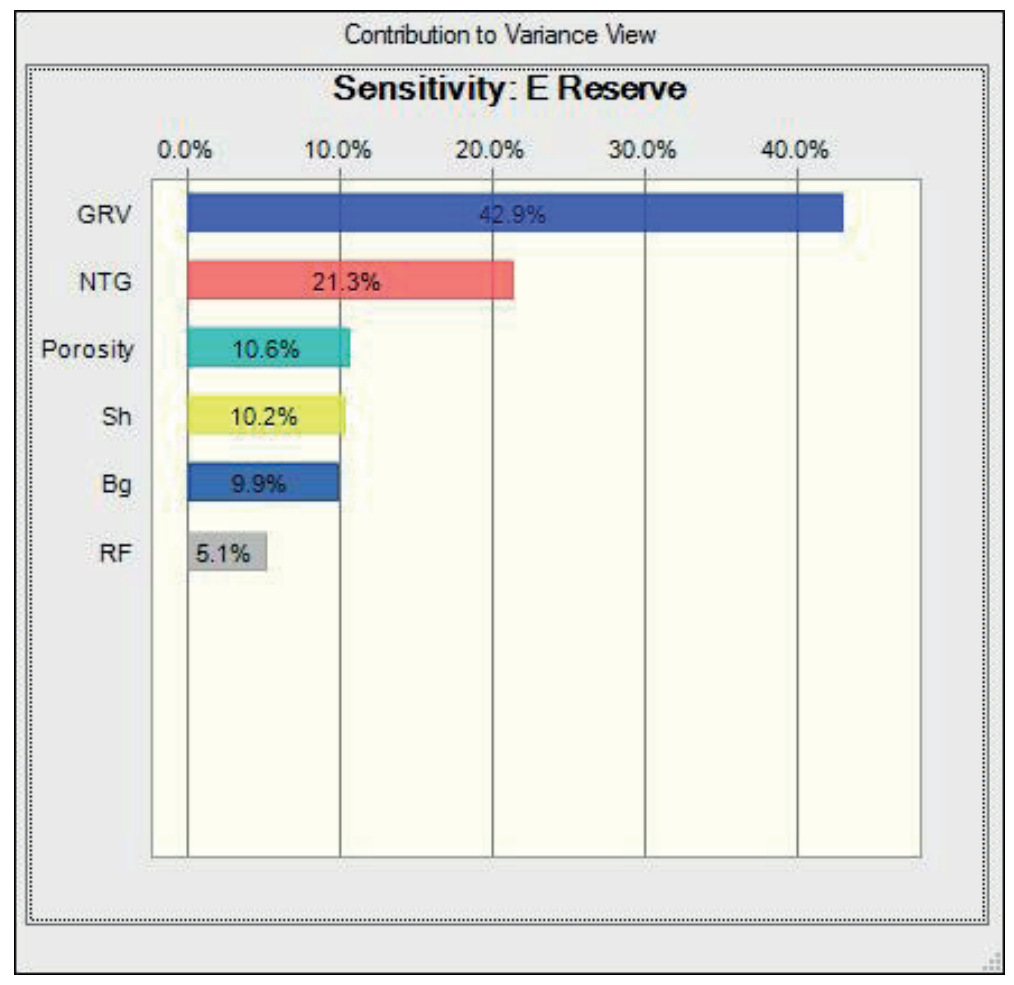

Fig. 15. The sensitivity impact on the E reserve simulation. Explanations as in Figure 13

\section{CONCLUSIONS}

This study was able to quantify the uncertainty for the ultimate recovery of 'OWA' onshore marginal field using the Monte Carlo simulation method. The coefficient of variability of about 15\%,19\% and $20 \%$ for B1, D and $\mathrm{E}$ respectively indicated that there is a very low level uncertainty of reserve estimation based on the distributions of input parameters. The sensitivity analysis of each parameter was measured for each simulation in their decreasing range of certainty.

A lead was identified west to the field from the depth structural maps which might boost reserves. Though a very small reserve, other deciding factors being equal, the 'OWA' marginal field can be developed and properly managed for positive yield on investment.

Many thanks to the Department of Petroleum Resources (DPR), Waltersmith Petroman Oil Limited and Degeconek Nigeria Limited.

\section{REFERENCES}

Avbovbo A.A., 1978. Tertiary Lithostratigraphy of the Niger Delta. American Association of Petroleum Geologists Bulletin, 62, 295-300.

Awotiku O.I., 2011. Quantification of Uncertainty and Risks for Developing Marginal Fields in the Niger Delta. The Department of Petroleum Engineering, African University of Science and Technology [M.Sc. thesis, unpublished].

Bickel J.E. \& Bratvold R.B., 2008. From uncertainty quantification to decision making in the oil and gas industry. Energy Exploration \& Exploitation Journal, 26, 311-325.

Doust H. \& Omatsola E., 1990. Niger Delta. [in:] Edwards J.D. \& Santogrossi P.A. (eds.), Divergent/passive Margin Basins, AAPG Memoir, 48, American Association of Petroleum Geologists, Tulsa, 239-248.

Egbogah E.O., 2011. Onshore/Marginal Field Developments: Challenges, Opportunities and Prospects for the Future. [Presented at:] 2011 SPE Annual Oloiribi Lecture and Energy Forum, Lagos, Nigeria.

Kulke H., 1995. Nigeria. [in:] Kulke H. (ed.), Regional Petroleum Geology of the World. Part II: Africa, America, Australia and Antarctica, Gebrüder Borntraeger, Berlin, 143-172.

Short K.C. \& Stauble J., 1967. Outline geology of the Niger Delta. American Association of Petroleum Geologists Bulletin, 5, 761-779.

Tuttle M., Charpentier R. \& Brownfield M., 1999. The Niger Delta Petroleum System: Niger Delta Province, Nigeria, Cameroon, and Equatorial Guinea, Africa. United States Geologic Survey. 